\title{
New limits on doubly charged bileptons from CERN LEP data and the search at future electron-positron and electron-photon colliders
}

\author{
E. M. Gregores \\ Instituto de Física Teórica, Universidade Estadual Paulista, 01405-900 São Paulo, SP, Brazil \\ and Department of Physics, University of Wisconsin, Madison, Wisconsin 53706 \\ A. Gusso \\ Instituto de Física Teórica, Universidade Estadual Paulista, 01405-900 São Paulo, SP, Brazil \\ and Instituto de Física Corpuscular-C.S.I.C., Universitat de València, E-46071 València, Spain \\ S. F. Novaes \\ Instituto de Física Teórica, Universidade Estadual Paulista, 01405-900 São Paulo, SP, Brazil
}

(Received 13 December 2000; published 30 May 2001)

\begin{abstract}
We show that the accumulated CERN LEP-II data taken at $\sqrt{s}=130-206 \mathrm{GeV}$ can establish more restrictive bounds on doubly charged bilepton couplings and masses than any other experiment so far. We also analyze the discovery potential of a prospective linear collider operating in both $e^{+} e^{-}$and $e \gamma$ modes.

DOI: 10.1103/PhysRevD.64.015004

PACS number(s): 14.80.-j, 12.60.-i, 13.88.+e
\end{abstract}

\section{INTRODUCTION}

Bileptons are bosons carrying double leptonic numbers that are predicted by some extensions of the standard model such as the $S U(15)$ based grand unified theory (GUT) [1], 3-3-1 model [2], and left-right symmetric models [3]. They can either be scalar or vector particles with different charges, i.e., neutral, singly, or doubly charged. In the 3-3-1 model, the symmetry breaking $S U(3)_{L} \times U(1)_{X} \rightarrow S U(2)_{L} \times U(1)$ is responsible for the appearance of vector bileptons, while in the left-right model, based on $S U(2)_{L} \times S U(2)_{R}$ $\times U(1)_{B-L}$, the bileptons correspond to the doubly charged scalar bosons present in the symmetry-breaking sector.

Bileptons can participate in a large variety of processes, both at low and high energies. However, no signal has yet been found, and bounds on their masses and couplings could be obtained from the analysis of lepton number violating processes [4], and muonium-antimuoniuon $(M-\bar{M})$ conversion $[5,6]$ experiments. The existing mass limits for both singly and doubly charged bosons $[5,7]$ are all model dependent. These bounds still allow the existence of low-mass bileptons with a small coupling constant. The limits from highenergy experiments [6] such as $e^{+} e^{-}$collisions are, in principle, less restrictive than the low-energy bounds.

In this paper we explore the production of doubly charged bileptons in both $e^{+} e^{-}$and $e \gamma$ colliders, looking for the most promising signature for identifying these particles: an isolated same sign, planar, and $p_{T}$ balanced two muon (antimuon) event $[6,8,9]$. We made a model independent analysis, working in the context of a general effective $S U(2)_{L}$ $\times U(1)_{Y}$ Lagrangian that couples bileptons to leptons [6]:

$$
\begin{aligned}
\mathcal{L}= & \lambda_{1} \bar{l}^{c} i \sigma_{2} l L_{1}+\tilde{\lambda}_{1} \bar{e}^{c} e \widetilde{L}_{1}+\lambda_{2} \bar{l}^{c} \gamma^{\mu} e L_{2 \mu} \\
& +\lambda_{3} \bar{l}^{c} i \sigma_{2} \vec{\sigma} l \cdot \vec{L}_{3}+\text { H.c. },
\end{aligned}
$$

where $l=\left(e_{L}, \nu_{L}\right)$ are left-handed $S U(2)_{L}$ lepton doublets, and $e=e_{R}$ are right-handed charged singlet leptons. The charge conjugated fields are defined as $\bar{l}^{c}=\left(l^{c}\right)^{\dagger} \gamma^{0}=$ $-l^{T} C^{-1}$. The subscript of the bilepton fields $L=1,2,3$ indicates whether they are singlet, doublet, or triplet under $S U(2)_{L}$. The terms concerning the doubly charged bileptons can be written as

$$
\begin{aligned}
\mathcal{L}^{--}= & \tilde{\lambda}_{1}^{i j} \bar{e}_{i}^{c} P_{R} e_{j} \tilde{L}_{1}^{--}+\lambda_{2}^{i j} \bar{e}_{i}^{c} \gamma^{\mu} P_{R} e_{j} L_{2 \mu}^{--} \\
& +\sqrt{2} \lambda{ }_{3}^{i j} \bar{e}_{i}^{c} P_{L} e_{j} L_{3}^{--}+\text {H.c. }
\end{aligned}
$$

where $e_{i}$ represent the charged leptons with flavor indices $i, j=1,2,3$, and $P_{R(L)}=\left(1 \pm \gamma_{5}\right) / 2$ are the helicity projectors. We will consider here only flavor diagonal bilepton couplings since very restrictive bounds are imposed by lowenergy experiments when flavor violation can take place $[4,6]$. These couplings will also be considered real to avoid $C P$ violating processes.

\section{LIMITS FROM LEP}

The whole CERN $e^{+} e^{-}$collider LEP program has been very successful. They were able to achieve both energy and luminosity beyond the values initially expected. However, no evidence for new physics has yet been found. From the nonobservation of pair produced bileptons model independent lower limits on the masses of these particles can be established to be close to $100 \mathrm{GeV}$. However, the present most stringent bounds on the mass and coupling of doubly charged bileptons come from the results of muonium-antimuonium conversion experiments [5]. For flavor diagonal couplings, these measurements require that the ratio of the bilepton coupling and its mass must satisfy $\widetilde{\lambda_{1}} / M_{B}<0.20 \mathrm{TeV}^{-1}(90 \%$ C.L.),$\quad \lambda_{2} / M_{B}<0.27 \mathrm{TeV}^{-1} \quad(95 \% \quad$ C.L. $)$, and $\lambda_{3} / M_{B}$ $<0.14 \mathrm{TeV}^{-1}$ (90\% C.L.). 

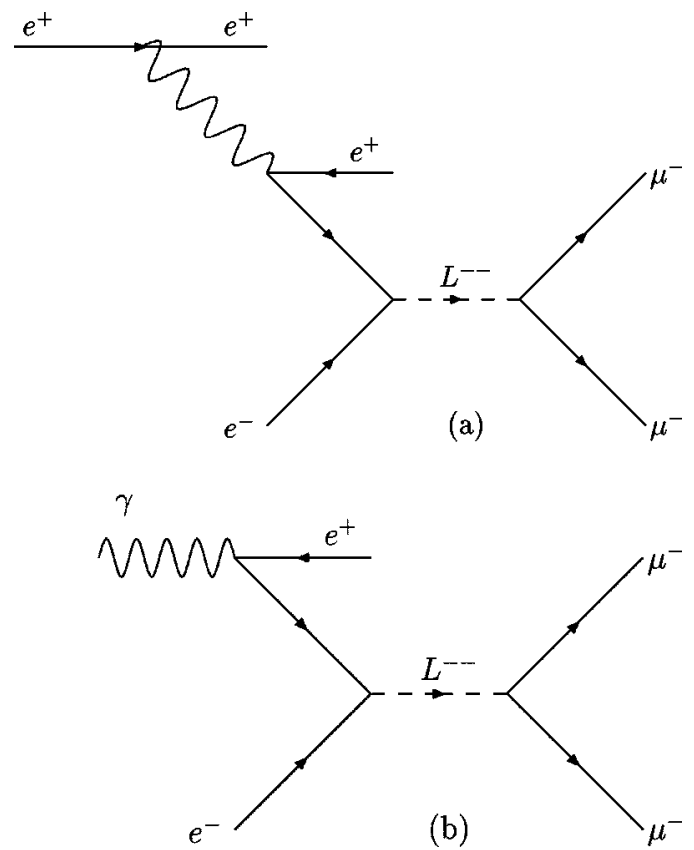

FIG. 1. Main contribution to the processes (a) $e^{+} e^{-} \rightarrow \mu^{ \pm} \mu^{ \pm}$ and (b) $e^{-} \gamma \rightarrow \mu^{-} \mu^{-}$.

We found that these limits can be overridden for the mass range kinematically accessible at the LEP collider. The occurrence of a high-energy event presenting just a $p_{T}$ balanced coplanar pair of same-sign leptons, notably muons or antimuons, would be a striking evidence for the presence of a bilepton. Since there is no standard model background for this kind of process, the observation of one such dimuon event would already constitute an important step towards the bilepton discovery. From the nonobservation of such an event a 90 (95) \% C.L. upper bound on the values of $\lambda$ and $M_{B}$ can be obtained based on the predicted number of 2.3 (3.0) events.

This signature results predominantly from the process depicted in Fig. 1(a), and its cross section has been evaluated in the equivalent particle approximation [10]. The deviation from the exact calculation is expected to be small since this process is dominated by events where the incident particle is scattered at a very small angle. The relevant cross section is given by

$$
\begin{aligned}
& \sigma\left(E_{e^{+}}, \hat{s}\right)_{e^{+} e^{-} \rightarrow \mu^{-} \mu^{-}} \\
& \quad=\int_{x_{\min }}^{1} d x F_{e^{+}}^{e^{-}}\left(x, E_{e^{+}}\right) \sigma(\hat{s})_{e^{-} e^{-} \rightarrow \mu^{-} \mu^{-},}
\end{aligned}
$$

where $F_{e^{+}}^{e^{-}}\left(x, E_{e^{+}}\right)$is the equivalent electron distribution function of the initial positron. It gives the probability that an

TABLE I. LEP integrated luminosities at different energies. The luminosity at $\sqrt{s}=133 \mathrm{GeV}$ is the weighted average of the luminosities obtained at 130 and $136 \mathrm{GeV}$.

\begin{tabular}{lcccccccccc}
\hline \hline$\sqrt{s}(\mathrm{GeV})$ & 133 & 161 & 172 & 183 & 189 & 192 & 196 & 200 & 202 & 206 \\
$\mathcal{L}(\sqrt{s})\left(\mathrm{pb}^{-1}\right)$ & 22 & 42 & 41 & 217 & 678 & 113 & 313 & 328 & 155 & 800 \\
\hline \hline
\end{tabular}
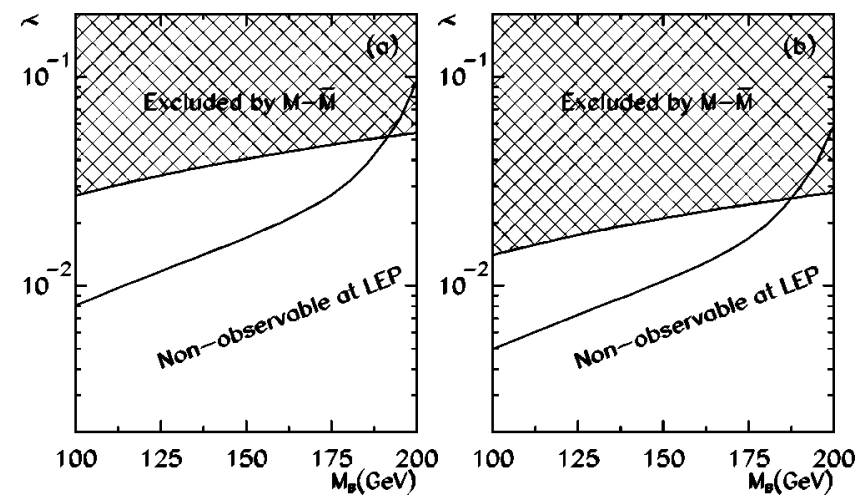

FIG. 2. Bilepton exclusion plot in the $\left(M_{B}, \lambda\right)$ plane for LEP data. (a) limits on $\lambda_{2}$ (95\% C.L.); (b) limits on $\lambda_{3}$ (90\% C.L.). See comments in Sec. II for the limits on $\tilde{\lambda_{1}}$.

electron with energy $E_{e^{-}}=x E_{e^{+}}$is emitted from a positron beam with energy $E_{e^{+}}$. The same holds true for the positron contents of the electron. This distribution is [11]

$$
\begin{aligned}
F_{e^{+}}^{e^{-}}\left(x, E_{e^{+}}\right)= & \frac{1}{2}\left\{\frac{\alpha}{2 \pi}\left[\ln \left(\frac{E_{e^{+}}}{m_{e}}\right)^{2}-1\right]\right\}^{2}\left(\frac{1}{x}\right) \\
& \times\left(\frac{4}{3}+x-x^{2}-\frac{4}{3} x^{3}+2 x(1+x) \ln x\right) .
\end{aligned}
$$

The cross section for the subprocess $e^{-} e^{-} \rightarrow \mu^{-} \mu^{-}$is

$$
\sigma(\hat{s})=S \frac{\lambda^{4} \hat{s}}{24 \pi\left[\left(\hat{s}-M_{B}^{2}\right)^{2}+M_{B}^{2} \Gamma^{2}\right]},
$$

where $\Gamma=G \lambda^{2} M_{B} /(8 \pi)$ is the $s$-channel resonance width for a bilepton of mass $M_{B}, \hat{s}=x s$ is the subprocess squared center of mass energy, $S=3,1,12$ and $G=3,1,6$ for $\tilde{L}_{1}^{--}, L_{2 \mu}^{--}$, and $L_{3}^{--}$, respectively.

We present in Table I the integrated luminosities for the different LEP energies we used in our calculations [12]. The total number of expected events (pairs of muons or antimuons with total invariant mass $M_{B}$ ) is calculated considering the luminosities obtained at center-of-mass energies that are larger than the bilepton mass, i.e.,

$$
\begin{aligned}
N\left(M_{B}\right)= & 2 \sum_{i} \Theta\left(\sqrt{s_{i}}-M_{B}\right) \mathcal{L}\left(\sqrt{s_{i}}\right) \\
& \times \int_{\hat{s}=4 m_{\mu}^{2}}^{s_{i}} d \hat{s} \sigma\left(\sqrt{s_{i}} / 2, \hat{s}\right)_{e^{+}} e^{-} \rightarrow \mu^{-} \mu^{-},
\end{aligned}
$$

where $N\left(M_{B}\right)$ is the number of expected events with mass $M_{B}$, and $\mathcal{L}\left(\sqrt{s_{i}}\right)$ assumes the values in Table I. The factor 2

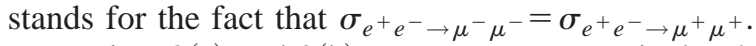

In Figs. 2(a) and 2(b) we present, respectively, the limits on $L_{2 \mu}^{--}$and $L_{3}^{--}$coupling constant that are imposed by the LEP data as a function of the bilepton mass. The solid line corresponds to $2.7 \mathrm{fb}^{-1}$ of integrated luminosity collected at energies ranging from $130 \mathrm{GeV}$ up to $206 \mathrm{GeV}$ by the four 


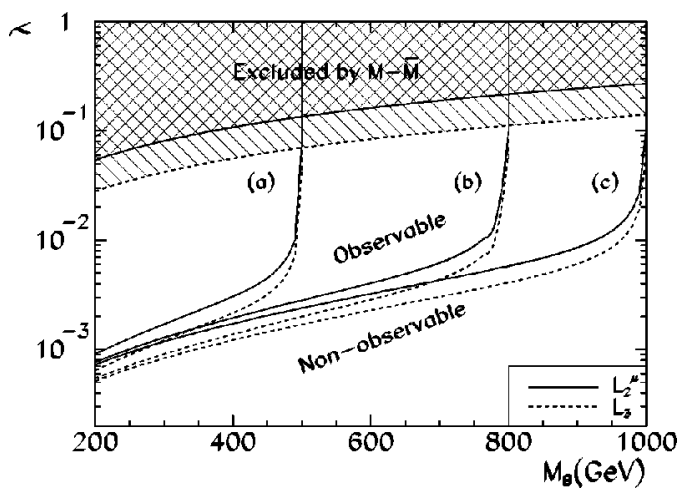

FIG. 3. Discovery region in the $\left(M_{B}, \lambda\right)$ plane at $95 \%$ C.L., assuming $\mathcal{L}=500 \mathrm{fb}^{-1}$ and $\sqrt{s}=500$ (a), 800 (b), and 1000 (c) $\mathrm{GeV}$, for a future $e^{+} e^{-}$linear collider.

LEP Collaborations. We also show the exclusion areas in the $\lambda-M_{B}$ parameter space imposed by $M-\bar{M}$ conversion experiments. The limits from the LEP data on $\widetilde{\lambda_{1}}$, and the region already excluded by $M-\bar{M}$ conversion, corresponding to $\tilde{\lambda}_{1} / M_{B}<0.20 \mathrm{TeV}^{-1}$ are not shown in Fig. 2 but can be readily obtained from the limits for $\lambda_{3}$ using the fact that $\tilde{\lambda_{1}}=\sqrt{2} \lambda_{3}$. In order to obtain these results we have taken into account an efficiency of $90 \%$ for dimuon reconstruction and a geometric acceptance of $|\cos \theta|<0.9$. This was a conservative choice for the geometric acceptance since we assumed the L3 value and all other experiments have larger acceptances. The maximum allowed values of $\lambda / M_{B}$ at LEP are smaller than those obtained from muonium-antimuonium conversion by a factor of 2 for most of the bilepton mass range.

\section{SEARCH AT FUTURE LINEAR COLLIDERS}

\section{A. Electron-positron collider}

Amid the efforts that are under way to determine the potentiality of proposed linear colliders to discover new physics [13], studies on bilepton searches have been carried out mostly for the $e^{-} e^{-}$collider operation mode $[6,9,14]$. We concentrate here on the potential of the $e^{+} e^{-}$and $e \gamma$ operation modes to discover doubly charged bileptons. We have assumed some representative values for the energy and luminosity of the new electron-positron machine [15], namely $\sqrt{s}=500,800$, and $1000 \mathrm{GeV}$ with $\mathcal{L}=500 \mathrm{fb}^{-1}$. This integrated luminosity is expected to be achieved in one or two years of the collider operation.

We evaluated the $e^{+} e^{-}$operation mode in a similar way as we did for LEP. In Fig. 3, we plot the 95\% confidence level discovery region in the $\lambda-M_{B}$ parameter space. In this figure, we also plotted the region of the parameter space already excluded by muonium-antimuonium conversion experiments. We considered a $90 \%$ dimuon reconstruction efficiency. We did not consider a particular value for the geometric acceptance, since we estimated that a $5^{\circ}$ aperture in the beam pipe region would not lead to more than a $2 \%$ event loss.

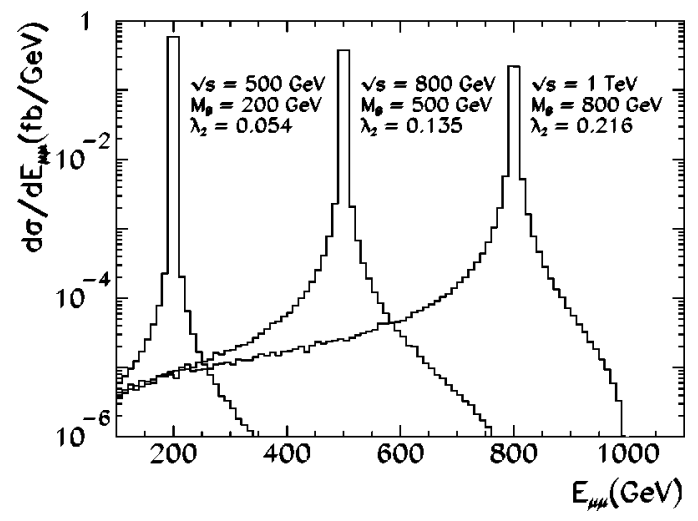

FIG. 4. Cross sections for the $\mu^{ \pm} \mu^{ \pm}$production in $e^{+} e^{-}$colliders as a function of their total invariant mass $E_{\mu \mu}$.

If bileptons are indeed observed, and profusely produced, it will be possible to determine their mass, and to learn whether they are vector or scalar particles. In Fig. 4, we present the expected dimuon invariant mass differential cross section, for some specific values of the vector bilepton coupling, which shows the resonant peak on the bilepton mass. We have chosen for $\lambda_{2}$ the maximum value allowed by the present $M-\bar{M}$ limits, i.e., $\lambda_{2}=0.27 M_{B}$.

The discrimination between vector and scalar can be done based on the fact that the cross section for the process $e^{-} e^{-} \rightarrow \mu^{-} \mu^{-}$changes with the polarization of the initial electron beams according to which of the three kinds of bileptons participates in the reaction. The discrimination can also be done using the fact that a vector bilepton leads to an angular distribution of the final state muons that is different from the angular distribution that would be observed if bileptons were scalars. The dependences on the initial beam polarizations $\left(P_{1}\right.$ and $\left.P_{2}\right)$ are shown in Table II, as well as the dependence on the angular distribution of the produced muons.

A discrimination based on the initial beam polarization is very promising since in the next generation of linear colliders one expects up to $80 \%$ of polarization for the electron beam. Assuming that the electron emitted from the positron beam is kept unpolarized $\left(P_{2}=0\right)$, we can see that, as the polarization of the electron beam $\left(P_{1}\right)$ increases, the cross section for the process mediated by $\widetilde{L}_{1}^{--}$increases while the cross section for the process mediated by $L_{3}^{--}$decreases. On the other hand, the insensitivity to $P_{1}$ would indicate the presence of a vector bilepton, as can be seen from Table II. However, if $\widetilde{L}_{1}^{--}$and $L_{3}^{--}$get mixed, the dependence on $P_{1}$ can be too small $\left(\widetilde{\lambda_{1}}=\sqrt{2} \lambda_{3}\right)$ to be observed, and it would

TABLE II. Angular and polarization dependence for $e^{+} e^{-}$ $\rightarrow \mu^{-} \mu^{-}$( $\theta^{*}$ is the C.M. angle $)$.

\begin{tabular}{lcc}
\hline \hline & Polarization dependence & Angular dependence \\
\hline$\tilde{L}_{1}^{--}$ & $\left(1+P_{1}\right)\left(1+P_{2}\right)$ & none \\
$L_{2 \mu}^{--}$ & $1-P_{1} P_{2}$ & $1+\cos ^{2} \theta^{*}$ \\
$L_{3}^{-}$ & $\left(1-P_{1}\right)\left(1-P_{2}\right)$ & none \\
\hline \hline
\end{tabular}



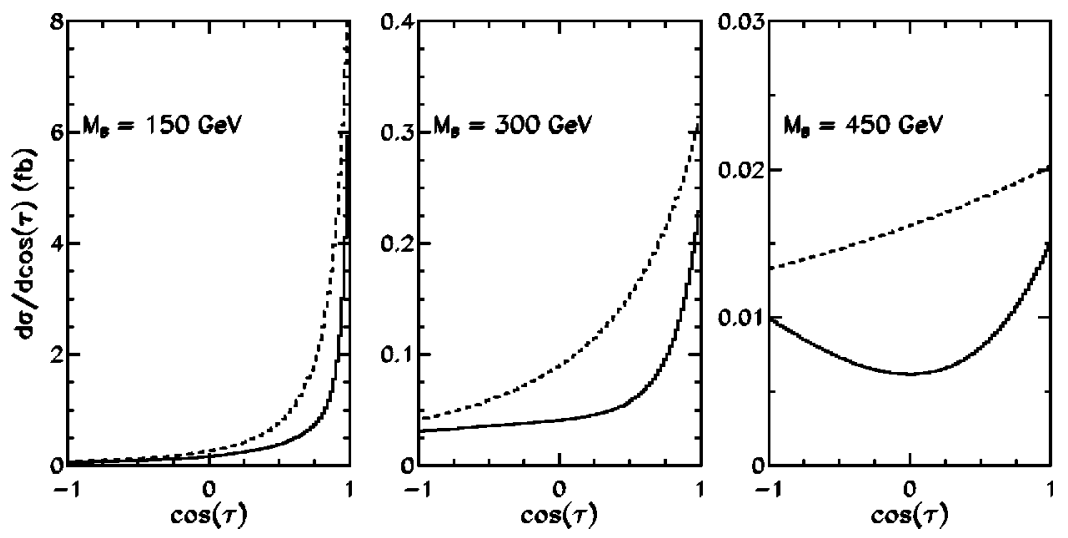

FIG. 5. Angular distribution in laboratory frame at $\sqrt{s}=500 \mathrm{GeV}$. The solid (dashed) line corresponds to vector (scalar) bileptons.

not be possible to distinguish between vectors and scalars by looking at the electron beam polarization dependence of the cross section.

The alternative would be to examine the angular distribution of the final-state muons. In Fig. 5 we can see the difference between the angular distribution of reactions mediated by scalar and vectorial bileptons at $\sqrt{s}=500 \mathrm{GeV}$. However, when the bilepton mass is relatively small, like for a 150$\mathrm{GeV}$ bilepton depicted in Fig. 5, the angular distributions for vector and scalar bileptons are quite similar. In this case, to distinguish vector from scalar particles it will require the knowledge of the bilepton mass with good accuracy. Fortunately, as can be seen from Fig. 5, the smaller the bilepton mass the bigger is the production cross section. This may help to make the identification task easier since more events are expected in this case for a given value of the coupling constant.

\section{B. Electron-photon collider}

Projects for future linear colliders include the possibility to transform the initial $e^{+} e^{-}$machine into an $e \gamma$ or $\gamma \gamma$ collider with comparable energy and luminosity [16]. In these machines, a highly energetic photon beam is generated by Compton backscattering low-energy photons emitted by a laser. The high-energy backscattered photons are then made to collide with the opposite incoming beam. For a nonpolarized collider, the backscattered photon distribution function is given by [17],

$$
f_{e}^{\gamma}(x)=\frac{2}{\sigma^{0}}\left[\frac{1}{1-x}+1-x-4 r(1-r)\right],
$$

where $x$ is the fraction of the electron momentum carried by the photon, $r=x /[y(1-x)]$, and

$$
\sigma^{0}=\left(2-\frac{8}{y}-\frac{16}{y^{2}}\right) \ln (y+1)+1+\frac{16}{y}-\frac{1}{(y+1)^{2}},
$$

where $y \approx 15.3 E_{B} \omega_{0}$, with the parent electron energy $E_{B}$ expressed in $\mathrm{TeV}$, and the laser energy $\omega_{0}$ in $\mathrm{eV}$. A maximum value $y=4.8$ is usually adopted to avoid electron regeneration through pair production. This is the $y$ value we used in our analysis.
In the e $\gamma$ collider, bileptons would be produced as a $s$-channel resonance through the diagram depicted in Fig. 1(b). Similarly to the treatment we employed for $e^{+} e^{-}$colliders, we also assumed here that the positron escapes unobserved down the beam pipe. The cross section for the process $e^{-} \gamma \rightarrow \mu^{-} \mu^{-}$in the equivalent particle approximation is given by

$$
\begin{aligned}
\sigma_{e^{-}} & \rightarrow \mu^{-} \mu^{-} \\
& =\int_{x_{1}^{\min }}^{x^{\max }} \int_{x_{2}^{\min }}^{1} d x_{1} d x_{2} f_{e}^{\gamma}\left(x_{1}\right) F_{\gamma}^{e}\left(x_{2}\right) \hat{\sigma}_{e^{-} e^{-} \rightarrow \mu^{-} \mu^{-}},
\end{aligned}
$$

where $f_{e}^{\gamma}\left(x_{1}\right)$ is given by Eq. (7), and $F_{\gamma}^{e}\left(x_{2}\right)$ is the distribution function of the equivalent electron carrying a fraction $x_{2}$ of the photon energy,

$$
F_{\gamma}^{e}\left(x_{2}\right)=\frac{\alpha}{\pi}\left[x_{2}^{2}+\left(1-x_{2}\right)^{2}\right] \ln \frac{E_{\gamma}}{m_{e}} .
$$

We also determined the potential of the $e \gamma$ collider to discover bileptons considering the observation of a single event. It has been assumed that the luminosity of the $e \gamma$ mode will be comparable to its parent $e^{+} e^{-}$mode. In Fig. 6 we plot the $95 \%$ confidence level discovery region in the $\lambda-M_{B}$ parameter space. In this figure, we also plotted the region of the parameter space already excluded by muoniumantimuonium conversion experiments. We can see that an $e \gamma$

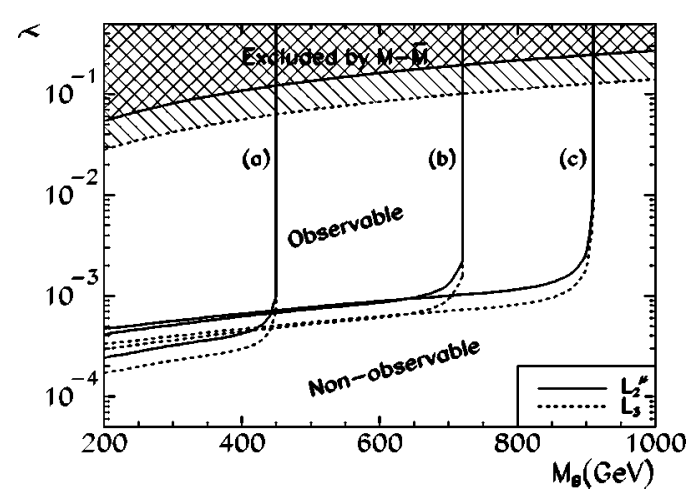

FIG. 6. The same as Fig. 3 for the $e \gamma$ mode of the linear collider. 


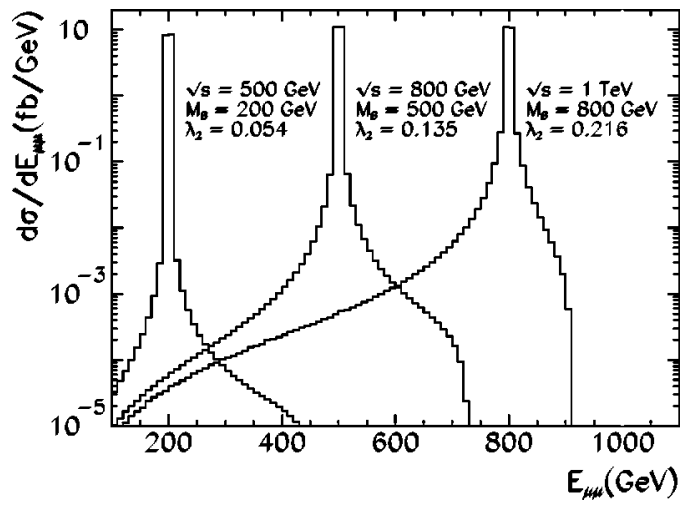

FIG. 7. Cross sections for the $\mu^{ \pm} \mu^{ \pm}$production in $e \gamma$ colliders as a function of their total invariant mass $E_{\mu \mu}$.

collider is more efficient for the search of bileptons than the $e^{+} e^{-}$collider. This is expected since the laser backscattering mechanism [Fig. 1(b)] is capable of generating a large amount of hard photons when compared with the usual bremsstrahlung subprocess [Fig. 1(a)].

If the bileptons are discovered and a fairly large amount of events is collected, we can use the same procedure, based on the electron polarization and angular distribution of finalstate muons, to determine their mass and spin. In Fig. 7, we present the expected event distribution as a function of the muon (or antimuon) pair invariant mass. As in Fig. 4 the value of $\lambda$ is the maximum allowed by the present $M-\bar{M}$ limits, i.e., $\lambda_{2}=0.27 M_{B}$. The angular distribution follows the same pattern as the one presented in Fig. 5.

\section{DISCUSSIONS AND CONCLUSIONS}

In this paper we have shown that new and more stringent limits on the coupling constants and masses of doubly charged bileptons can be obtained from LEP-II data. As for the limits from $M-\bar{M}$ conversion, our results apply to the case of flavor-diagonal bilepton couplings. These stringent bounds result from the fact that the integrated luminosity collected by the LEP Collaborations at energies above 189 $\mathrm{GeV}$ is very large. In order to obtain the new limits on $\lambda$ and $M_{B}$ we have applied the equivalent particle approximation method. It is expected that an exact tree level calculation of the cross section would lead to only minor corrections to our results [10]. A few percent uncertainty in the cross sections translates into even smaller corrections to the curves in Figs. 2,3 , and 6 because the cross sections are proportional to $\lambda^{2}$. Our results suggest that an accurate analysis taking into account detailed LEP detectors and data sample properties will be able to establish bounds on bilepton couplings and masses that overcome the present ones.

We have also shown that the construction of high-energy linear accelerators could lead to a large increase in the sensitivity to the doubly charged bileptons. If these new particles are in the reach of these machines, a quite evident
TABLE III. Values of $\lambda_{2}$ that lead to the expected number of three events in the case $\mathcal{L}=500 \mathrm{fb}^{-1}$.

\begin{tabular}{ccccc}
\hline \hline$M_{B}$ & \multicolumn{2}{c}{$\lambda_{2} \times 10^{4}(500 \mathrm{GeV})$} & \multicolumn{2}{c}{$\lambda_{2} \times 10^{4}(1 \mathrm{TeV})$} \\
\hline & $e^{-} e^{-}$ & $e \gamma$ & $e^{-} e^{-}$ & $e \gamma$ \\
\hline 200 & 2.50 & 2.40 & 1.46 & 4.72 \\
300 & 0.96 & 3.22 & 1.36 & 5.55 \\
400 & 0.60 & 4.21 & 1.23 & 6.64 \\
500 & 0.11 & & 1.10 & 7.73 \\
\hline \hline
\end{tabular}

resonance peak should be observed without the need to scan the collision energy. The sensitivity to bileptons of the general purpose $e \gamma$ collider is of the same order of magnitude as the $e^{-} e^{-}$operating mode. In order to compare both operation modes we updated the results presented in Ref. [8] on a bilepton search in $e^{-} e^{-}$colliders, to the luminosity of 500 $\mathrm{fb}^{-1}$ considered here. The reanalysis is straightforward and is based on the fact that the cross section for the process $e^{-} e^{-} \rightarrow \mu^{-} \mu^{-}$is proportional to the square of the bilepton coupling and the limits on $\lambda$ will be proportional to $\sqrt{1 / \mathcal{L}}$, where $\mathcal{L}$ is the collider luminosity. In Table III we compare the limits on $\lambda_{2}$ for the two kinds of colliders operating at $\sqrt{s}=0.5$ and $1 \mathrm{TeV}$, with $\mathcal{L}=500 \mathrm{fb}^{-1}$. As it would be expected, the $e^{-} e^{-}$mode is, in general, more sensible than the e $\gamma$ mode. This advantage may be, however, illusory. The instantaneous luminosity delivered by the $e^{-} e^{-}$collider is expected to be half that of the $e^{+} e^{-}$with same beam characteristics because of the antipinch effect. This fact slightly diminishes the advantage of the $e^{-} e^{-}$mode over the $e \gamma$ mode for bilepton production. By comparing Fig. 3 with the values in Table III, we can see that the direct $e^{+} e^{-}$mode is clearly less sensitive than the other two operation modes. Nevertheless, it is much more sensitive than the present experiments.

In conclusion, a collider operating at $e^{+} e^{-}$or $e \gamma$ modes is quite sensitive to the resonant production of doubly charged bileptons. If bileptons are indeed observed in these machines we will probably be able to get a lot of information about them. Later, their properties could be thoroughly studied using the $e^{-} e^{-}$mode operating at $\sqrt{s}=M_{B}$.

\section{ACKNOWLEDGMENTS}

E.M.G. is grateful to the University of Wisconsin for its kind hospitality. A.G. would like to thank the hospitality at the Instituto de Fisica Corpuscular (IFIC-UV) where part of this work was carried out. S.F.N. is grateful to Fermilab for its hospitality. This work was supported by Conselho Nacional de Desenvolvimento Científico e Tecnológico ( $\mathrm{CNPq})$, by Fundação de Amparo à Pesquisa do Estado de São Paulo (FAPESP), by Programa de Apoio a Núcleos de Excelência (PRONEX), and by Fundação para o Desenvolvimento da UNESP (FUNDUNESP). 
[1] P. H. Frampton and B.-H. Lee, Phys. Rev. Lett. 64, 619 (1990).

[2] F. Pisano and V. Pleitez, Phys. Rev. D 46, 410 (1992); P. H. Frampton, Phys. Rev. Lett. 69, 2889 (1992).

[3] J. C. Pati and A. Salam, Phys. Rev. D 10, 275 (1974); R. N. Mohapatra and J. C. Pati, ibid. 11, 566 (1975).

[4] P. Frampton and D. Ng, Phys. Rev. D 45, 4240 (1992); H. Fujii, S. Nakamura, and K. Sasaki, Phys. Lett. B 299, 342 (1993); G. K. Leontaris, K. Tamvakis, and J. D. Vergados, Phys. Lett. 162B, 153 (1985); U. Bellgardt et al., Nucl. Phys. B299, 1 (1988); J. D. Bjorken and S. Weinberg, Phys. Rev. Lett. 38, 622 (1977).

[5] L. Willmann et al., Phys. Rev. Lett. 82, 49 (1999); H. Fujii, Y. Mimura, K. Sasaki, and T. Sasaki, Phys. Rev. D 49, 559 (1994); D. Chang and W.-Y. Keung, Phys. Rev. Lett. 62, 2583 (1989).

[6] F. Cuypers and S. Davidson, Eur. Phys. J. C 2, 503 (1998); N. Leporé, B. Thorndyke, H. Nadeau, and D. London, Phys. Rev. D 50, 2031 (1994).

[7] M. B. Tully and G. C. Joshi, Phys. Lett. B 466, 333 (1993); P. H. Frampton and M. Harada, Phys. Rev. D 58, 095013 (1998).

[8] F. Cuypers and M. Raidal, Nucl. Phys. B501, 3 (1997).

[9] P. H. Frampton and A. Rasin, Phys. Lett. B 482, 129 (2000).

[10] V. N. Baier, V. S. Vadin, and V. A. Khoze, Nucl. Phys. B65, 381 (1973); M.-S. Chen and P. Zerwas, Phys. Rev. D 12, 187 (1975); A. Zembrzuski and M. Krawczyk, DESY HERA
Workshop, 1991, p. 617; I. F. Ginzburg and V. G. Serbo, Phys. Rev. D 49, 2623 (1994).

[11] T. Sjöstrand, Comput. Phys. Commun. 82, 74 (1994).

[12] DELPHI Collaboration, P. Abreu et al., Phys. Lett. B 485, 45 (2000); LEP Working Group for Higgs Boson Searches, P. Bock et al., CERN-EP-2000-055, to appear in 35th Rencontres de Moriond on Electroweak Interactions and Unified Theories, Les Arcs, France, 2000; LEP Working Group on Four Jets, M. A. Bizouard et al., CERN-OPEN-98-008; L3 Collaboration, M. Acciarri et al., Phys. Lett. B 485, 85 (2000); OPAL Collaboration, G. Abbiendi et al., ibid. 476, 256 (2000); ALEPH Collaboration, R. Barate et al., ibid. 469, 287 (1999).

[13] See, for instance, M. Peskin, SLAC-PUB-8288, hep-ph/9910521.

[14] M. Raidal, Phys. Rev. D 57, 2013 (1998).

[15] American Linear Collider Working Group, J. Bagger et al., hep-ex/0007022. See also information available at http:// www.desy.de/schreibr/physics-wg.html and http:// www.slac.stanford.edu/xorg/ilc-trc/ilc-trchom.html

[16] V. I. Telnov, in 'Proceedings of 4th International Workshop on Linear Colliders 1999,' hep-ph/9910010; I. F. Ginzburg, Nucl. Phys. B (Proc. Suppl.) 82, 367 (2000).

[17] D. L. Borden, D. A. Bauer, and D. O. Caldwell, SLAC-PUB5715, 1992; Report No. UCSB-HEP-92-01, 1992; Phys. Rev. D 48, 4018 (1993). 